\title{
CDISC SEND Tumor Findings Results Terminology
}

National Cancer Institute

\section{Source}

National Cancer Institute. CDISC SEND Tumor Findings Results Terminology. NCI

Thesaurus. Code C88025.

The terminology that includes concepts relevant to the Clinical Data Interchange Standards Consortium (CDISC) Standard for the Exchange of Non-clinical Data (SEND) tumor findings character results. 